\title{
Plurilinguismo e politiche linguistiche
}

L'esempio del CIEBP

\section{Tullio Telmon}

\section{(2) OpenEdition \\ Journals}

Edizione digitale

URL: http://journals.openedition.org/esp/3708

DOI: $10.4000 /$ esp.3708

ISSN: 2532-0319

\section{Editore}

Centre d'Information sur l'Éducation Bilingue et Plurilingue

\section{Edizione cartacea}

Data di pubblicazione: 1 juin 2019

Paginazione: 7-18

ISSN: 1127-266X

\section{Notizia bibliografica digitale}

Tullio Telmon, «Plurilinguismo e politiche linguistiche», Éducation et sociétés plurilingues [Online], 46 |

2019, Messo online il 01 février 2021, consultato il 01 mars 2021. URL: http://

journals.openedition.org/esp/3708 ; DOI: https://doi.org/10.4000/esp.3708

Questo documento è stato generato automaticamente il 1 mars 2021.

(C) CIEBP 


\section{Plurilinguismo e politiche linguistiche}

L'esempio del CIEBP

Tullio Telmon

Une première version de ce article est parue in

Lingue Antiche e Moderne, 7, 2019.

\section{Atteggiamenti contraddittori}

1 L'archetipo della maledizione babelica, dalla quale sarebbero derivate la moltiplicazione e la confusione delle lingue, pur essendo la dimostrazione implicita della fattualità storica dei plurilinguismi, si è imposto, attraverso i millenni e attraverso i secoli, come rappresentazione concreta di un ruolo sostanzialmente negativo che la pluralità di lingue svolgerebbe nel consorzio umano. Da esso, variamente declinato e adattato ai diversi momenti storici e alle diverse temperie culturali, discendono gli atteggiamenti di ostilità nei confronti di ciò che è linguisticamente diverso e ad esso è strettamente collegato lo stereotipo negativo consistente nel ritenere l'educazione plurilingue nociva per la crescita cognitiva: fino ad oltre la metà del secolo appena trascorso, insegnare altre lingue, accanto e in complementarietà con quella materna, era considerata nel migliore dei casi un'eccentricità; in ogni caso, pedagogisti, educatori, insegnanti e anche insigni glottologi non esitavano a sostenere che un'educazione bilingue (e a fortiori un'educazione plurilingue) costituisse per i fanciulli un rischio fortissimo di disordine e confusione mentale (Tabouret-Keller 2011). In Italia, tale atteggiamento fu indubbiamente rafforzato, a partire dalla raggiunta unità politica, dalla convinzione, da parte degli uomini politici e degli intellettuali, che in assenza di una parallela unità linguistica l'unità politica si rivelasse troppo fragile. Di qui, l'ostilità nei confronti dei dialetti e di qui, la forte convergenza su di un'ideologia monolingue da parte della scuola e dei suoi operatori. Di qui, le istanze puristiche, il rifiuto di ogni contaminazione derivante dal contatto con altre lingue, la diffidenza nei confronti dei cambiamenti. 
2 Ma, si sa, il cambiamento è connaturato con l'essenza stessa della lingua, e i contatti tra lingue diverse fanno parte della normalità, sia all'interno di una certa comunità linguistica sia nell'individuo che ne è membro (Py 2005: 74). Per quanto riguarda l'Italia, inoltre, l'adesione all'Europa unita, accompagnata ad un effettivo apprendimento della lingua italiana da parte di quel $92 \%$ di popolazione che, a detta di De Mauro (1963), era esclusivamente dialettofona nel 1861, sembra ora avere portato ad un graduale abbandono dell'ideologia monolingue e ad una sua sostituzione con una ideologia plurilingue, polarmente opposta. Un'ideologia che, si noti bene, sembra investire l'intero Occidente e accompagnarsi con i fenomeni di globalizzazione e di modernizzazione ${ }^{1}$ che contraddistinguono il momento attuale.

Ma, si chiede ancora Py (2005: 74), sarà davvero così? L'attuale apparente regresso dell'ideologia monolingue è legato ad un'evoluzione reale delle rappresentazioni ordinarie del linguaggio, o non è altro che un discorso alla moda, politicamente corretto, vale a dire ad un insieme di formule prefabbricate e convenzionali (ad altri stereotipi, in sostanza), che non impegnano davvero il locutore che le enuncia? I segnali sono contraddittori, ma non depongono affatto a favore le tendenze puristiche che, ogni volta che nascono polemiche su questio-ni linguistiche, emergono con l'invocazione di prese di posizione da parte di istanze preposte alla garanzia e al mantenimento della pretesa purezza della lingua.

\section{Alcune iniziative}

4 A dimostrazione di un atteggiamento ideologico completamente mutato si possono citare le numerose associazioni, organizzazioni e i numerosi centri e osservatori che hanno fatto del plurilinguismo il loro principale oggetto di ricerca, di studio, di divulgazione. Citerò qui, in estrema sintesi, alcuni di quelli a me più noti. In ordine cronologico:

- OLSI - Osservatorio Linguistico della Svizzera Italiana http://www.ti.ch/olsi, attivo dal 1991. Dir.: Prof. Bruno Moretti;

- CIP > CEIP - Centro Internazionale sul Plurilinguismo (Università degli Studi di Udine) https://www.uniud.it/it/ateneo-uniud/ateneo-uniud-organizzazione/altre-strutture/ centro-internazionale, attivo dal 1993. Dir.: Prof. Renato Oniga. Diffonde anche un bollettino: CEIPnews, n. 1, aprile 2017 (a cura di Paolo Driussi);

- OEP - Observatoire Européen $\mathrm{du}$ Plurilinguisme http:// www.observatoireplurilinguisme.eu/, attivo dal 2005. Pres.: Christian Tremblay. Anche questo Osservatorio diffonde una 'lette-ra di informazione'; cfr. la più recente: Lettre d'information de l'OEP, $\mathrm{N}^{\circ} 71$ (sept.-oct. 2017).

-LEM - Langues d'Europe et de la Méditerranée - http://www.portal-lem.com. Associazione LEM-Italia http://www.associazionelemitalia.org/, attivo dal 2008. Fondatore: Henri Giordan; Resp. It.: Giovanni Agresti.

5 A monte di tutta questa attività è sicuramente da mettere in rilievo l'autorevolezza e l'importanza della politica europea che, malgrado alcuni aspetti contraddittori, non può non trovare la sua ragione di essere proprio nel plurilinguismo ad essa connaturato. Ne è testimonianza la Carta Europea del Plurilinguismo, elaborata dall'Observatoire Européen du Plurilinguisme e destinata ad essere sottoposta al voto 
del Consiglio d'Europa e al Parlamento europeo. Questi i dodici punti della sua versione abbreviata:

1. Le plurilinguisme est inséparable de l'affirmation d'une Europe politique;

2. La diversité des langues assure la pluralité et la richesse des représentations;

3. Le plurilinguisme est une liberté;

4. Le plurilinguisme est un moyen d'affirmer la pérennité des entités nationales et infranationales, lieux privilégiés d'exercice de la citoyenneté;

5. Le plurilinguisme est une source fondamentale du sentiment de citoyenneté européenne;

6. Le plurilinguisme est une des réponses au "choc des civilisations" et aux diverses formes d'hégémonie politique, culturelle et économique;

7. Le plurilinguisme est un élément essentiel de l'innovation scientifique;

8. Le droit à la langue et à la diversité linguistique et culturelle ne se divise pas;

9. Le plurilinguisme est un gage de progrès économique dans un monde pacifié;

10. Tout travailleur doit avoir le droit de travailler dans la langue du pays où il vit;

11. Les systèmes éducatifs doivent offrir une éducation plurilingue;

12. Les médias doivent permettre l'expression des cultures multiples.

\section{Dal CMIEB al CIEBP}

6 Come, dopo la prima guerra mondiale, le speranze e i progetti di pace furono affidati alla creazione della Società delle Nazioni, così, negli anni successivi alla seconda guerra mondiale, nasce un movimento, quello delle "città gemellate", con obiettivi identici e con la speranza che ciò che non era riuscito alla Organizzazione delle Nazioni Unite (scongiurare e prevenire lo scatenarsi di una guerra mondiale), potesse invece riuscire facendo leva sui comuni, entità presumibilmente meno legate a condizionamenti nazionalistici e più vincolate alle realtà dei problemi concreti - presumibilmente simili in ogni paese - della vita quotidiana dei cittadini.

7 Fu così che nacque, nel 1957, la "Fédération Mondiale des Villes Jumelées". Sei anni prima, era stato creato un "Conseil des Communes d'Europe", di ispirazione europeista. La nuova Federazione nasce dunque con l'intento di allargare il campo di azione a tutto il globo, compresi i paesi del cosiddetto "Patto di Varsavia". Cosa, quest'ultima, che venne fortemente censurata dal Cce (Chombard-Gaudin 1992). Ciò che accomunò l'uno e l'altro organismo fu la consapevolezza dell'importanza della questione linguistica. Ma mentre il Cce fu propenso a scegliere il bilinguismo reciproco anglo-francese (inglese come seconda lingua per tutti i francesi; francese per tutti i britannici), ponendosi così dei limiti piuttosto severi verso l'apertura e la diffusione dei propri obiettivi, l'opzione della FMVJ fu per il plurilinguismo: più vaga, forse, ma certo molto meno ristretta. La successiva evoluzione delle politiche europeiste sembrarono infatti dare ragione alla scelta plurilingue della FMVJ. E infatti, nel 1968, dalla Fédération fu originato, proprio per dare maggior vigore al versante linguistico dei propri obiettivi, il CMIEB: Centre Mondial d'Information sur l'Éducation Bilingue. Tra i promotori, il più attivo fu il sindaco della città di Aosta, Giulio Dolchi, che qualche anno dopo, da Presidente della Regione autonoma della Valle d'Aosta, portò la sua amministrazione ad assumere il patronato del nuovo organismo, assicurando così le risorse minime necessarie per il suo funzionamento. Il primo presidente del CMIEB fu André Martinet, che mantenne l'incarico finché, ormai ottantacinquenne, lasciò la presidenza alla propria allieva 
Andrée Tabouret-Keller, rimasta in carica fino all'autunno 2018 e ora sostituita da chi scrive. Mentre la Fédération mondiale des Villes Jumelées diventava dapprima Fédération mondiale des cités unies et villes jumelées, poi Fédération Mondiale des Cités unies e infine si fondeva, nel 2004, con l'anglofona International Union of Local Authorities (IULA) e con Metropolis, per deventare "Cités et gouvernements locaux unis" (United Cities and Local Governments), grazie al lungimirante provvedimento di Dolchi, il CMIEB imboccava la strada di una sempre maggiore autonomia rispetto all'ente che lo aveva creato. Nel 1996 aggiunse, alla propria intitolazione, anche l'aggettivo "plurilingue", e nel 2004 dismise il "mondiale". Il CMIEB era divenuto CIEBP, un Centro dotato di un proprio statuto e di un Consiglio di amministrazione, composto da diciassette membri, che si riunisce due volte all'anno e che ogni tre anni convoca l'Assemblea degli iscritti per il rinnovo delle cariche. È dotato di un suo sito (http:// www.ciebp.org) e produce una rivista, Éducation et Sociétés plurilingues / Educazione e Società plurilingui, con uscita semestrale.

\section{Obiettivi}

Mentre nella prima fase della sua attività gli scopi del Centro erano quelli dettati dalla Federazione che lo aveva creato e consistevano, fondamentalmente, nel preconizzare l'acquisizione precoce di una seconda lingua e nell'incoraggiare questo tipo di acquisizione servendosi della rete di relazioni, scambi e gemellaggi della FMVJ, dopo il distacco dalla Fédération agli scopi sopra citati si aggiunsero altri obiettivi, più vicini al mondo della ricerca linguistica e sociolinguistica e, al tempo stesso, più attenti all'evolversi delle politiche linguistiche in atto sia nella realtà europea sia nel più vasto panorama delle lingue del mondo. Tra gli obiettivi prioritari riportati nello Statuto, citeremo qui quello di studiare le modalità di realizzazione dell'educazione plurilingue, quello di dare testimonianza delle sperimentazioni e delle realizzazioni di educazione plurilingue, sia nella dimensione pedagogica sia in quella dei rapporti con la società, e quello di difendere la libertà dei mezzi di espressione a tutti i livelli, dalla più umile delle parlate locali alle lingue "universalizzate".

\section{Azioni principali}

Pur nella limitatezza dei propri mezzi finanziari, il Centro cerca di raggiungere i propri scopi attraverso tre principali azioni: l'organizzazione di convegni internazionali su temi legati alla pluralità linguistica; l'organizzazione, due volte all'anno, di una conferenza pubblica su temi e argomenti legati all'attualità della ricerca linguistica, sociolinguistica, geolinguistica, glottodidattica, di linguistica educazionale; infine, la pubblicazione regolare di una rivista semestrale, Éducation et Sociétés plurilingues / Educazione e Società plurilingui.

\section{Congressi internazionali}

Nell'ultimo decennio, sono stati tre i Convegni organizzati dal CIEBP. Tutti e tre si sono svolti in Valle d'Aosta (il secondo, parte in Valle d'Aosta e parte a Torino) e tutti e tre hanno avuto, come è logico attendersi da un organismo i cui interessi sono internazionali per definizione, una forte impronta di internazionalità. Il primo si è 
svolto nel 2006 a Saint Vincent, Aosta e Cogne, ed ha avuto per titolo "Dove va la Dialettologia?". Il secondo è stato il frutto di una collaborazione con la SLI (Società di Linguistica Italiana), della quale chi scrive era, in quel momento, il presidente; si è svolto nel 2011 ad Aosta, Bard, Torino, ed aveva per titolo "Coesistenze linguistiche nell'Italia pre- e postunitaria". Il terzo, svoltosi nel 2016 ad Aosta presso l'Università della Valle d'Aosta (che ne era partner) è stato dedicato a tematiche percezionali ed ha avuto per titolo "Langues faibles".

\section{I 'Samedi du CIEBP'}

11 Il secondo tipo di azione condotta e sviluppata dal CIEBP è quella che viene ormai chiamata famigliarmente "Les samedi matin du CIEBP". Si tratta di conferenze pubbliche (due all'anno). Tali conferenze sono state ospitate, a partire dal 1997 e fino al 2015, dalla Maison des Sciences de l'Homme di Parigi. Dal 2015, le conferenze hanno trovato ospitalità presso la saletta delle Edizioni dell'Harmattan, in Rue des Écoles a Parigi. I conferenzieri sono scelti in modo da offrire un panorama più vario possibile delle situazioni bi- e plurilingui in tutto il mondo, tanto nella scuola quanto in altri settori della vita sociale.

Dal 2007, le stesse o altre conferenze vengono offerte anche ad Aosta, dove vengono ospitate dalla Università della Valle d'Aosta, con la quale il CIEBP ha stipulato una convenzione di collaborazione.

\section{Alcuni esempi}

Il primo incontro dei "Samedi matin" è avvenuto il 10 maggio 1997. Nel corso di esso, Jean Salles-Lousteau ha parlato de L'enseignement des langues régionales en France.

Di tutti e tre i Convegni internazionali sono stati realizzati e pubblicati gli Atti; cfr. Raimondi-Revelli 2007; Telmon-Raimondi-Revelli 2012 e Revelli-Tabouret-Keller-Varro 2017. Complessivamente, è stata realizzata sino ad oggi oltre una quarantina di incontri, con ricercatori, educatori, responsabili di progetti, registi, su argomenti quali, ad es., il trilinguismo del Lussemburgo, la presenza di alunni extracomunitari nelle classi delle elementari, le reti di informazione sul plurilinguismo, il linguaggio dei giovani, gli spazi delle imprese in regioni transfrontaliere, l'intercomprensione, il bilinguismo in famiglia, l'insegnamento integrato della lingua e della disciplina, ecc.

Una delle più recenti conferenze, per portare un altro esempio è stata tenuta da Giovanni Ruffino il 25 marzo 2017. Il titolo era Accogliere uomini, accogliere parole. Lampedusa: una storia esemplare di ieri e di oggi; dato il notevole interesse dell'argomento, la conferenza è stata replicata anche ad Aosta il successivo $1^{\circ}$ dicembre.

\section{La rivista}

La terza azione che il CIEBP conduce per il raggiungimento dei propri obiettivi consiste, come si è detto, nella pubblicazione di una rivista semestrale dal titolo bilingue Éducation et sociétés plurilingues / Educazione e società plurilingui. Il primo numero porta la data di dicembre 1996; l'ultimo pubblicato è il 45, del dicembre $2018^{3}$. 


\section{Le vicende del frontespizio}

Il titolo e il frontespizio hanno subito, negli anni, una serie di piccole modifiche, formali e di sostanza. Per fare un esempio, mentre nel primo numero i caratteri del titolo erano in maiuscoletto, a partire dal numero 2 (settembre 1997) l'intera intitolazione è stata fatta in caratteri maiuscoli. Con il numero 15 (dicembre 2003), la rivista si è dotata di un numero di ISSN (1127-266 X), mentre soltanto con il numero 20 (giugno 2006) si è provveduto a correggere, nella parte italiana del titolo, l'aggettivo "plurilingui", che in precedenza era erroneamente indicato al singolare ("plurilingue"). Soltanto con il numero 28 (giugno 2010) si è infine provveduto a dotare dei corretti accenti acuti le parole francesi "Éducation" e "Sociétés". Resta a mio avviso una anomalia, consistente nell'uso improprio dell'apostrofo, anziché dell'accento, sulla parola italiana "Società". Vedremo se anche a questa mancanza verrà posto rimedio.

\section{Redazione e Comitato di lettura}

Responsabile della pubblicazione è Gianmario Raimondi, di Aosta, mentre le responsabili della redazione sono Gabrielle Varro (Parigi) e Marisa Cavalli (Aosta). La rivista è dotata di un Comitato di lettura, composto da Marisa Cavalli, Stefano Corno, Gilbert Dalgalian, Christine Deprez, Federica Diémoz, Luisa Giovanni, Yannick Lefranc, Bruno Moretti, Gianmario Raimondi, Luisa Revelli, Matteo Rivoira, Ana-Isabel Ribera, Gérald Schlemminger (corrispondente), Andrée Tabouret-Keller, Claude Truchot, Gabrielle Varro.

Nei 20 anni di vita, si sono avvicendati ventotto membri del Comitato di lettura.

Hanno scritto per ESP 346 diversi autori, provenienti da trentotto diversi stati dei cinque continenti.

\section{Le rubriche di ESP}

Queste le rubriche, che, con la sola eccezione dell'Editoriale (quasi sempre firmato dalla Presidente del CIEBP, Andrée Tabouret-Keller), non necessariamente ricorrono tutte in ogni numero:

1. Editoriale

2. Storia e Società

3. Vallee d'Aosta

4. Didattica e Insegnamento bi-/plurilingue

5. Esperienze e Ricerche

6. Discussioni

7. Testimonianze

8. Recensioni

9. Informazioni

Vediamone partitamente le caratteristiche. 


\section{Gli editoriali di ATK} proposte di procedure ludiche, resoconti di atelier, sperimentazioni, attività varie, uso di materiali della letteratura orale popolare, "portfolio delle lingue" come strumento didattico, esercizi di ortofonia, riflessioni sugli errori interlinguistici, sulla lingua dei segni, sulla gestione del repertorio linguistico. E ancora: insegnamento bilingue spagnolo-francese in Spagna, didattica della letteratura, letteratura come didattica linguistica, proposte pedagogiche per il bilinguismo nell'isola della Réunion, il progetto VRAL sull'intercomprensione, il "curriculum minimo" per un primo contatto con l'italiano, competenze plurilingui e pluriculturali, rappresentazioni mentali e rappresentazioni sociali delle lingue di apprendimento, abilità linguistiche e analisi della pubblicità, "passaggi dinamici" da una lingua all'altra nella scuola materna, ecc.

A partire dal numero 13 (dicembre 2002), una speciale sottorubrica è stata dedicata, in particolare, alle esperienze didattiche e di ricerca sul plurilinguismo attuate in Valle d'Aosta.

\section{Esperienze e ricerche}

Pur sconfinando talvolta con quella precedente, questa rubrica è quella a cui sono affidati in genere i "pezzi" più teorici. Vengono accolti testi di ricercatori esperti e 
autorevoli, ma anche proposte di giovani studiosi alla pubblicazione del loro primo articolo scientifico.

Fin dal primo numero, vediamo articoli sul ruolo del bilinguismo nella competenza comunicativa (Titone), e poi, ad es., sul ruolo delle donne nelle politiche linguistiche familiari (Deprez), sull'immersione bilingue (Titone), sul "principio dello sforzo condiviso" nei conflitti di lingua (Weydt), su identità nazionali e sovranazionali nei giovani (Dorenlot), sulla lettura a voce alta in classe (Djouadi), sulle strategie di risoluzione di conflitti etnici e identitari in classi multietniche (Kucukcan), sull'educazione linguistica dei figli di immigrati a Torino (Mosca), sui tipi di saperi nell'insegnamento bilingue (Gajo), sul futuro delle lingue nel mondo globalizzato (Siguan), sul ruolo contraddittorio della scuola nella costruzione delle identità plurilingui (Gérin-Lajoie), sulle relazioni tra lingue di minoranza e identità (Raimondi), sul ruolo dell'arabo dialettale (Boukra), su glossodiversità e biodiversità (Dalgalian), su plurilinguismo e mercati (Etrillard), ecc.

\section{Discussioni}

31 Anche questa rubrica è partita assai per tempo, e si mostra molto utile e ricca di spunti interessanti. Riporto qui alcuni dei temi di discussione affrontati nel corso del tempo:

$\mathrm{N}^{\circ} 4$ e 5: critiche e rilievi alla nuova riforma delle lingue nella primaria (Dalgalian, Chéhab e Luc).

$\mathrm{N}^{\circ}$ 6: la sconfitta, in California, della Transitional Bilingual Education spagnolo-inglese (Dorenlot).

$\mathrm{N}^{\circ}$ 7: le scuole francesi all'estero devono essere esclusivamente francofone? (Vivet).

$\mathrm{N}^{\circ}$ 8: il dibattito su russo e bielorusso in Bielorussia (Fourse).

$\mathrm{N}^{\circ}$ 9: utilizzare il francese a lezione di inglese? (Benguigui-Mejia).

$\mathrm{N}^{\circ} 10$ : basta con i falsi cliché sul plurilinguismo (Hare).

$\mathrm{N}^{\circ}$ 13: critiche al modello ideologico della lingua unica (arabo) in Algeria (Benrabah).

$\mathrm{N}^{\circ} 14$ e 15 lingue dei giovani (Canobbio, Sayah).

$\mathrm{N}^{\circ} 19$ e 20: rappresentazioni linguistiche e malintesi nell'insegnamento plurilingue (Py, Lefranc, Py, Leralu).

$\mathrm{N}^{\circ} 22$ e 23: uso scolastico delle rappresentazioni sociali sulle lingue di minoranza (Verny, Forêt, Dompmartin-Normand).

$\mathrm{N}^{\circ}$ 27: Le lingue e le linguistiche sono politiche? (Lefranc).

$\mathrm{N}^{\circ} 35$ : quali effetti si possono prevedere dall'insegnamento in inglese nelle università francesi? (Truchot).

Stranamente, non ha avuto successo l'unico argomento che è stato, per così dire, lanciato dalla redazione: la valutazione del code switching e del code mixing nella prassi didattica dell'insegnamento di L2. I pochi interventi giunti in redazione si sono rivelati poco attinenti o superficiali; può darsi che, riproponendo l'argomento in modo mirato a specialisti del settore, si possano ottenere risultati migliori.

\section{Testimonianze}

Si tratta, soprattutto nei primi numeri, di informazioni su situazioni poco conosciute di forme di bi- o plurilinguismo in atto (Paesi baschi francesi, Belgio, Moldavia...) o di 
testimonianze di alunni di scuole in cui si praticano forme, anche diverse, di bilinguismo. plurilinguismo come oggetto di interesse, pare oggi un segnale di abbandono dell'ideologia monolingue: un'ideologia generalmente intrisa di nazionalismi, che si riflette, a livello di teoria linguistica, nell'ignoranza di una delle principali caratteristiche di ogni lingua naturale umana, consistente nella variabilità. Sul piano sociolinguistico, l'altra grossolana colpa di cui l'ideologia monolingue è gravata è la parallela ignoranza della complessa rete di varietà di codici di cui i repertori linguistici di ogni comunità sociale sono portatori.

41 Di più: in una ideologia monolingue la lingua viene intesa come entità autonoma $e$ omogenea, monolitica ed immobile, da proteggere da ogni contaminazione. Di qui, gli atteggiamenti puristici e rigidamente normativi che caratterizzano gli assertori di questa ideologia. diverse e tra livelli diversi della stessa lingua e la loro compresenza sia nella comunità sia nell'individuo. A giudicare dalle manifestazioni di pensiero che, sull'argomento, contraddistinguono il momento attuale, si direbbe dunque che l'ideologia plurilingue abbia ormai conquistato il sentire comune.

In chi, come me, ha vissuto e ha per professione registrato il mutamento di ideologia dominante, resta però un dubbio: siamo di fronte ad un'evoluzione reale delle rappresentazioni sociali del linguaggio o non piuttosto ad un discorso alla moda, politicamente corretto? Altrimenti detto: si tratta di consenso convinto o di consenso di superficie?

Difficile dare una risposta, anche perché la mia sensazione è che, in fatto di rappresentazioni sociali, di percezioni e di atteggiamenti non soltanto non esista mai 
un punto di arrivo, ma che tali fattori siano spesso soggetti a fenomeni di ciclicità, con ritorni inopinati a stadi che si credevano superati.

Non soltanto, ma data la contraddittorietà che, come da più parti è stato fatto rilevare, sembra uno dei tratti più costanti dei giudizi percettivi dei parlanti, non è da escludere che anche chi afferma di essere convintamente favorevole al plurilinguismo assuma poi, nella pratica, atteggiamenti tipici dell'ideologia monolingue.

Come quel professore di matematica, di cui ci parla Bernard Py (2005) che può essere, in quanto cittadino $\mathrm{o}$ in quanto genitore, un partigiano globalmente convinto dell'insegnamento bilingue, ma che non si riterrà in contraddizione con se stesso allorché preciserà che, in quanto insegnante di matematica, desidera che la sua disciplina sia insegnata in modo monolingue, perché ai suoi occhi la matematica è troppo complessa per potersi offrire il lusso di un insegnamento bi-lingue.

\section{BIBLIOGRAFIA}

CHOMBARD-GAUdin C. 1992. Pour une histoire des villes et communes jumelées, Vingtième Siècle, revue d'histoire, $\mathrm{n}^{\circ} 35$ (juill.-sept): 60-66.

http://www.persee.fr/doc/xxs_0294-759_1992_num_35_1_2565

DE MAURo T. 1963. Storia linguistica dell'Italia unita, Bari, Laterza.

Py B. 2005. Représentations langagières et malentendus dans l'en-seignement plurilingue, Éducation et Société plurilingues, 19 (déc.): 73-81.

RAIMONDI G., REVELli, L. (a cura di) ; 2007. La dialectologie aujourd'hui. Atti del Convegno interna-zionale “Dove va la dialettologia?", Saint-Vincent - Aosta - Cogne 21-24 settembre 2006, Alessandria, Edizioni dell'Orso.

ReVelli L., TABouret-Keller A., VARRO G. (a cura di). 2017. Langues faibles. Lingue deboli, ParigiTorino, L'Harmattan.

TABOURET-KeLLER A 2011. Le bilinguisme en procès, cent ans d'errance (1840-1940), Limoges, LambertLucas.

TELMON T., RAIMONDI G., REVELli L. (a cura di). 2012. Coesistenze linguistiche nell'Italia pre-e postunitaria. Atti del XLV Congresso Internazionale di Studi della Società di Linguistica Italiana (SLI), Aosta-Bard-Torino 26-28 settembre 2011, 2 voll., Roma, Bulzoni.

\section{NOTE}

1. A dire il vero, il risvolto sociolinguistico della modernizzazione sembra piuttosto guardare al monolinguismo come traguardo da raggiungere. Coerentemente con i principi di massimo profitto con il minimo sforzo, il fenomeno della modernizzazione porta infatti, generalmente, ad agire contro le lingue minori e a favore di quelle maggiori. 
2. Nel momento in cui questa comunicazione veniva letta, il 23 novembre 2017 in occasione del "Primo Colloquio Internazionale sul plurilinguismo", organizzato del Centro Internazionale sul Plurilinguismo e dall'Università di Udine, l'ultimo numero era in realtà il 42, del giugno 2017.

3. Va fatto notare, a questo proposito, che la prassi della stampa francese era in precedenza molto più tollerante nei confronti della mancata apposizione dell'accento sui caratteri maiuscoli. Credo che una maggiore attenzione sia ora determinata dall'uso del computer, che consente ora di ampliare di molto i segni disponibili in tastiera.

\section{RIASSUNTI}

Dans cet article, nous résumons certaines des initiatives les plus significatives concernant la recherche ou les projets portant sur le bilinguisme et le plurilinguisme depuis la fin du $20^{\text {ème }}$ et le début du $21^{\text {ème }}$ siècle. Nous serons particulièrement attentifs au CIEBP (Centre d'Information sur l'Education Bilingue et Plurilingue), né en 1968 et dont le premier président était André Martinet, à une époque où l'attitude de la pédagogie officielle envers l'éducation bilingue précoce était encore fortement négative. Le CIEBP faisait partie de la Fédération Mondiale des Villes Jumelées. Nous discuterons de ses objectifs, son développement, son organisation et ses accomplissements depuis ses débuts et jusqu'à nos jours.

In this article we review some of the most important initiatives dealing with the research or projects on bilingualism and plurilingualism, initiated between the end of the 20th and the beginning of the 21st century. We will specially concentrate on the CIEBP (Information Centre on Bilingual and Plurilingual Education), born in 1968 and whose first president was André Martinet, at a time when the attitude of official pedagogy towards early bilingual teaching was still strongly negative. We will discuss its aims, development, organization and achievements from its beginnings, when the CIEBP was part of the Twin Towns World Federation, to the present day.

\section{INDICE}

Mots-clés : bilinguisme, plurilinguisme, CIEBP, éducation plurilingue, congrès, revue Keywords : bilingualism, multilingualism, CMIEB, CIEBP, plurilingual education, congresses, journal

\section{AUTORE \\ TULLIO TELMON}

Université de Turin (Italie) 